\title{
A New Year and a New Liaison
}

This journal has emphasized that psychosomatic medicine is alive and well in the new millennium [1-4]. We believe that the psychosomatic message is more and more valid and timely in today's medicine. The constant growth in visibility of our journal (the 2000 impact factor increased to 2.37) is one of the many examples that could be provided. It thus comes as no surprise that we have established a liaison with a rapidly expanding association, the International College of Psychosomatic Medicine. In 2002 all ICPM members will receive a copy of our journal as part of their dues. This provides an additional reason for joining the ICPM for those who have not done it yet. Those who wish to do so should contact the ICPM Secretariat, c/o IPS, Kvibergsvagen 5, S-41505 Goteborg (Sweden).

Below we report a list of the experts who have supplemented the editorial board in reviewing the manuscripts submitted to Psychotherapy and Psychosomatics in 2001.

Both external referees and editorial board members have been requested to disclose any potential conflict of interest when revieweing a paper. The editor-in-chief did not have any conflict of interest in 2001.

\section{References}

1 Fava GA: Lipowski's legacy: The psychosomatic spirit. Psychother Psychosom 1999;68:1-2.

2 Sivik T: Psychosomatic medicine: Why fix it if it ain't broken? Psychother Psychosom 2000;69:178-180.

3 Ryff CD, Singer BH: Biopsychosocial challenges of the new millennium. Psychother Psychosom 2000;69:170-177.

4 Fava GA, Sonino N: Psychosomatic medicine. Psychother Psychosom 2000;69:184-197.

\section{Giovanni A. Fava \\ Editor-in-Chief \\ Psychotherapy and \\ Psychosomatics}

\author{
Tatjana Sivik \\ President \\ International College of \\ Psychosomatic Medicine
}

Arnetz B. (Uppsala, Sweden)

Balestrieri M. (Udine, Italy)

Battaglia M. (Milano, Italy)

Belaise C. (Bologna, Italy)

Blanchard E.B. (Albany, N.Y., USA)

Buske-Kirschbaum A. (Trier, Germany)

Cahill S. (Philadelphia, Pa., USA)

Coelho R. (Porto, Portugal)

Conti S. (Bologna, Italy)

Copeland P. (Salem, Mass., USA)

Costantini A. (Roma, Italy)

De Girolamo G. (Roma, Italy)

Delle Chiaie R. (Roma, Italy)

Fabbri S. (Providence, R.I., USA)

Facchinetti F. (Modena, Italy)

Fassino S. (Torino, Italy)

Favaro A. (Padova, Italy)

Ferguson E. (Nottingham, UK)

Gelenberg A.J. (Tucson, Ariz., USA)

Grabe H.J. (Stralsund, Germany)

Healy D. (Bangor, UK)

Honkalampi K. (Kuopio, Finland)
Hoyer J. (Dresden, Germany)

Jarrett R.B. (Dallas, Tex., USA)

Kaplan H. (Toronto, Ont., Canada)

Labbate L.A. (Charleston, S.C., USA)

Lazaratou H. (Athens, Greece)

Liakopoulou M. (Athens, Greece)

Lumley M.A. (Detroit, Mich., USA)

Mangelli L. (Bologna, Italy)

Mangweth B. (Innsbruck, Austria)

Manu P. (Glen Oaks, N.Y., USA)

Mayou R. (Oxford, UK)

Meyer T. (Berlin, Germany)

Mota Cardoso R. (Porto, Portugal)

Nierenberg A. (Boston, Mass., USA)

Ottolini F. (Modena, Italy)

Pariante C. (London, UK)

Picardi A. (Roma, Italy)

Pope H.G. (Belmont, Mass., USA)

Probst M. (Kortenberg, Belgium)

Pruessner J. (Montreal, Que., Canada)

Radanov B. (Zurich, Switzerland)

Rafanelli C. (Bologna, Italy)
Ruini C. (Bologna, Italy)

Simpson G. (Los Angeles, Calif., USA)

Sobrinho L. (Lisboa, Portugal)

Santonastaso P. (Padova, Italy)

Schnyder U. (Zurich, Switzerland)

Schoenfeld R. (Göteborg, Sweden)

Snaith P.H. (Leeds, UK)

Sonino N. (Padova, Italy)

Starr J.M. (Edinburgh, UK)

Stern R. (London, UK)

Stilley C.S. (Pittsburgh, Pa., USA)

Swinson R. (Hamilton, Ont., Canada)

Tomba E. (Bologna, Italy)

Toscano V. (Roma, Italy)

Tossani E. (Bologna, Italy)

Treasure J. (London, UK)

Van Moffaert M. (Gent, Belgium)

Vieta E. (Barcelona, Spain)

Wessely S. (London, UK)

Zimmermann C. (Verona, Italy)

\begin{tabular}{ll}
\hline KARGER & ○ 2001 S. Karger AG, Basel \\
Fax +41 61 306 12 34 & 0033-3190/02/0711-0001\$18.50/0 \\
$\begin{array}{l}\text { E-Mail karger@karger.ch } \\
\text { www.karger.com }\end{array}$ & $\begin{array}{l}\text { Accessible online at: } \\
\text { www.karger.com/journals/pps }\end{array}$
\end{tabular}

